\title{
The Educational Neuroscience Perspective of ABR and Lullabies: Setting Up an Infants Brain Development Study
}

\author{
Efthymios Papatzikis \\ Canadian University Dubai, U.A.E.
}

\begin{abstract}
Research has shown that Music is very important for children's development, having an impact from a very early age. There is a variety of psychological research evidence suggesting that infants when actively take part in music classes, they widen their positive social trajectory and follow an earlier enculturation development. They better exploit the 'music properties' (i.e. tempo, timbre, rhythm) which are intrinsic to communication between them and their parents, while they also enhance their communication skills in both linguistic and functional societal terms. Biological evidence suggest that they put very early at work their already specialised for music processing brain while enhancing and specializing even more their neuronal circuitry. Nevertheless, early years research rarely refers to subcortical structures, thus missing a fundamental scope and angle of this valuable knowledge. This paper proposes a protocol on that specific subject of the early years subcortical development, explaining why and how such an inquiry should and can be investigated with the help of music.
\end{abstract}

\section{Introduction}

Around the globe, we all the more see the intentional usage of music and sound to increasingly expand as a 'tool' of biopsychological intervention in the context of the prenatal and postnatal (perinatal) health care [1-11]. The fact that music and sound engagement perinatally has been proved to provide an element of positive socio-psychological and learning development [12-16] strengthens the aforementioned trend.

Following the neuroscience research path, investigations focusing on the early years of life have clearly shown that there are functional specialisations for music and sound processing in the human brain (for example [17]; see also example of rhythm in [18]). It has been more specifically suggested that early music training and involvement may be responsible for superior development of prelinguistic communicative gestures and social behavior [19] while early exposure to music in children as young as 4 years, has clearly shown to enhance the P2 auditory evoked brain potential (reflecting the post-synaptic activity of a specific neural process measured through EEG, peaking at about 200 milliseconds after the onset of an external stimulus) compared to children who were not exposed to music (see Figure 1).

Especially for the auditory system, which mostly matures during the first 6 months after birth [21], early music involvement is crucial. The cochlea and the auditory cortex in the temporal lobe are both affected early by the environment and care practices, thus making this first period of life the most critical time for the development of the neurosensory paths of the auditory apparatus [22]. For foetuses, infants and toddlers, early sound and music engagement (formal or informal alike, either occurring through sound/musical interactions between newborns and caregivers, or through the passive sound/music perception that foetuses experience in the womb) is crucial for their later cognitive development [19], [20], [23 - 25].

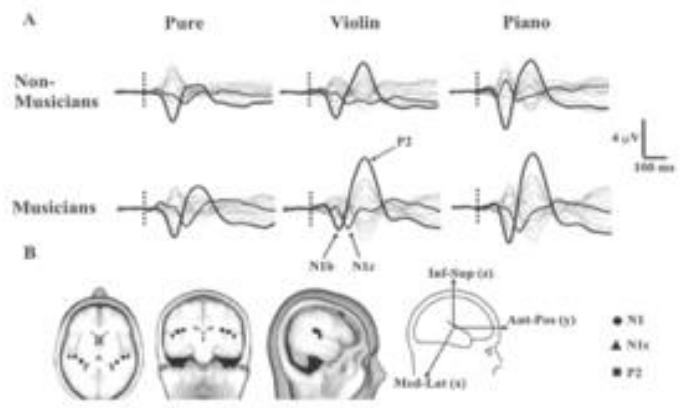

Figure 1. Upper Panel

EEG traces for all 28 channels evoked by the sine wave, violin and piano tones for musicians and non musicians. The dashed vertical line indicates onset of the tones. The $\mathrm{Cz}$ electrode is shown in bold and the T8 electrode is a dashed line. (Lower Panel) The locations of the regional sources for grand average data with a hemispheric symmetry constraint for the 
$\mathrm{N} 1 \mathrm{~b}, \mathrm{~N} 1 \mathrm{c}$ and the P2 components determined by BESA and superimposed on the average brain of BESA (reproduced directly by [20]).

\section{Early brainstem development and sound perception}

The impact of sound and music extends through our acoustical apparatuses (ears) and body to the whole brain, activating many of the latter's regions [26] (see Figure 2). One of these regions being involved in music/sound perception, analysis and decoding processes, especially in the early instances of development, is this one of the brainstem including both the hindbrain and midbrain encephalic parts [27], [28].

The brainstem starts structurally at the top of the spinal cord and is the most highly developed area of the baby's brain at birth. The brainstem is responsible for the most vital bodily functions including breathing, heartbeat, circulation, sucking and swallowing. Apart from these functions, it is crucially involved in sound perception as it accommodates the central auditory pathways, which extend from the cochlear nucleus to the auditory cortex. These auditory pathways are made to preprocess certain sound and music related qualities and characteristics like sound localisation and binaural interaction.

The inherent to the brainstem auditory pathways reach the midbrain and the auditory cortex through three main routes: the dorsal acoustic stria, the intermediate acoustic stria and the trapezoid body [29]. The brainstem auditory pathways are responsible for "the development of the tonotopic columns in the auditory cortex, [which].... are needed to receive, recognise and react to language, music and meaningful environmental sounds" [22].

Although the auditory system becomes fully functional at around 25 to 29 weeks gestational age, evidence has been found for an auditory evoked response at 16 weeks gestational age where the synapses of the VIII cranial nerve have been mostly functional [22], [30]. This is also when the development of the auditory system starts to get vulnerable to epigenetic processes, meaning that both endogenous (stimulation dependent) and exogenous (activity dependent) to the foetus's brain and in utero sound factors and elements may alter its structure and function. The auditory system development process critically extends until the 5-6 month of age, although it never stops up to the end of life [22].

\section{Problem statement}

It is already established that the brainstem is one of these important brain systems - sound-wise hugely developing during the perinatal period.
However, not much - to almost none - research has directly addressed this possible correlation that may exist between music education and its neuronal growth; in function and structure. How is this specific part of the subcortical brain region influenced from this non-invasive yet very powerful neuroplastic force that music imposes on the neuronal circuits of the specific brain structure?

Considering that the very first perinatal months of development are the most sensitive in epigenetic changes - that is, the changes that the environment induces to our biological and cognitive mechanisms it is crucial for research to provide solid evidence on the neuroanatomical and neurofunctional developmental changes of the brainstem as they occur through early sound and music exposure, especially when knowing that the latter are both closely connected and deeply embedded in our everyday lives from the very beginning.

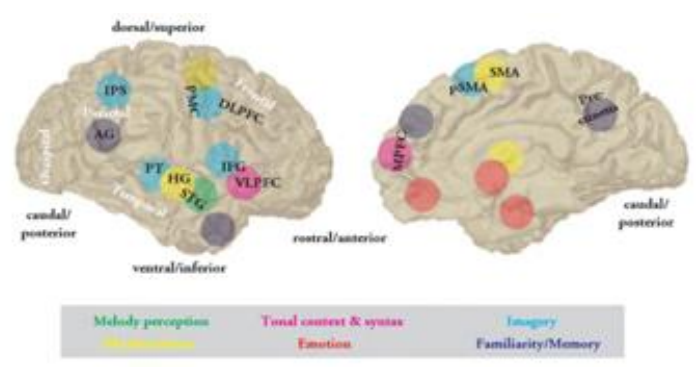

Figure 2. A highly schematised and simplified summary of brain regions involved in different facets of music psychological processes

On the left is the later view of the right cerebral hemisphere. A medial view of the right hemisphere is shown on the right. There is no intent to imply lateralisation of function in this portrayal. White lettering designates the different lobes. The coloured circles corresponde to the coloured labels in the box below the figure. AG: angular gyrus - DLPFC: dorsolateral prefrontal cortex - HG: Helsch's gyrus IFG: Inferior frontal gyrus - IPS: Intraparietal sulcus - MPFC: medial prefrontal cortex - PMC: premotor cortex - pSMA: pre-supplementary motor area - PT: planum temporale - SMA: supplementary motor area - STG: superior temporal gyrus - VLPFC: ventrolateral prefrontal cortex (reproduced from [33: 128]).

\section{Methods and research questions}

Wanting to provide a basic understanding on how music impacts the brain(stem) development in the early postnatal period, this project aims to collect data from a sample of babies, answering the following research question: Is there a direct correlation between specific musical features of lullabies (e.g. rhythm, 
timbre, structural complexity) and the infants' brainstem development when functionally measured in the first three weeks post delivery?

The infants' brainstem functional development will be directly provoked and measured in vivo, using music and more specifically lullabies as the intervention tool. The electroenchephalography (EEG) method, and particularly the Auditory Brainstem Response (ABR) technique (see Figure 3) will be employed in the first 40 days post delivery, measuring Auditory Evoked Potentials (AEPs) before and after the musical intervention (see Figure 4).

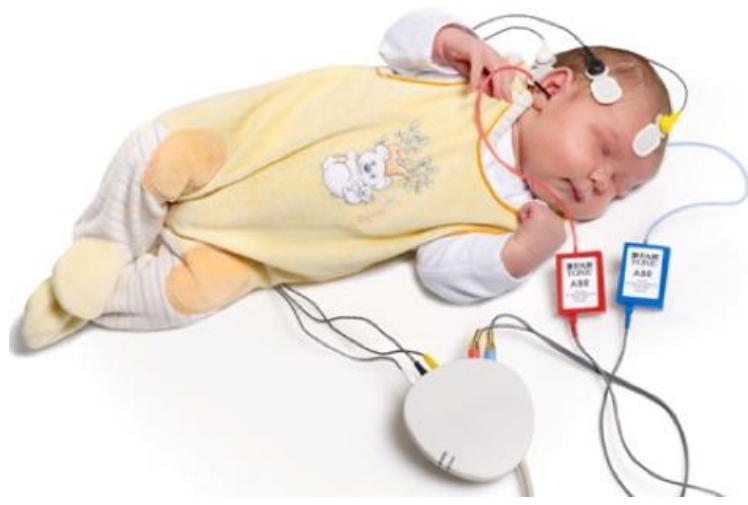

Figure 3. The auditory brainstem response (ABR) equipment and application

The Auditory Brainstem Response (ABR) is a particular EEG technique referring to the auditory brainstem responses "which can be measured using scalp electrodes that pick up electrical potentials generated by the synchronous activity of neural populations in the brainstem. Because these aggregate neural responses that can be recorded objectively and passively, they offer an excellent means to assess auditory function" [32].

Basic ABR protocols usually employ repeated clicks and sinusoidal tones to maximise the brainstem responses. However, these are not approximate in simulating behaviourally relevant and complex sounds which mostly exist outside of the lab, in the real life human environment and interactions (i.e speech, music etc.). Therefore, more complex stimuli were gradually adopted in the auditory neuroscience domain and the ABR registration process, forming the complex ABR registration technique or the $\mathrm{cABR}$. There is a plethora of complex ABR stimuli that are now used in relevant investigations, a full review of which can be found in the comprehensive ABR tutorial by Skoe and Kraus [32].

\section{Research rationale - Hypothesis}

In the early days and weeks of human development, specific stimuli, which are either delivered through the mother during the gestation period or - postnatally - in direct to the baby, may provide an efficient platform of advancement when correctly used as intervention tools.

Music can be easily employed as an intervention tool in this early developmental context, as it is not clinically invasive, while the primary sensorial system related to its perception and analysis (the acoustical apparatus and the brainstem, that is) is one of the first to be functional in the perinatal period of life. The fact that research has already found that "subcortical processing of sound is not hardwired" [32] makes the specific intervention tool a valuable one to study early brain development, as it could definitely pronounce structural and functional brain alterations during its implementation.

Based on the above, it is mainly hypothesise in this study that music and more specifically lullabies can evoke perinatally a more efficient brainstem development), suggesting also in extension that lullabies' proper inclusion in the early clinical and educational settings should be mandatory towards a later enhanced cortical development and sensitivity.

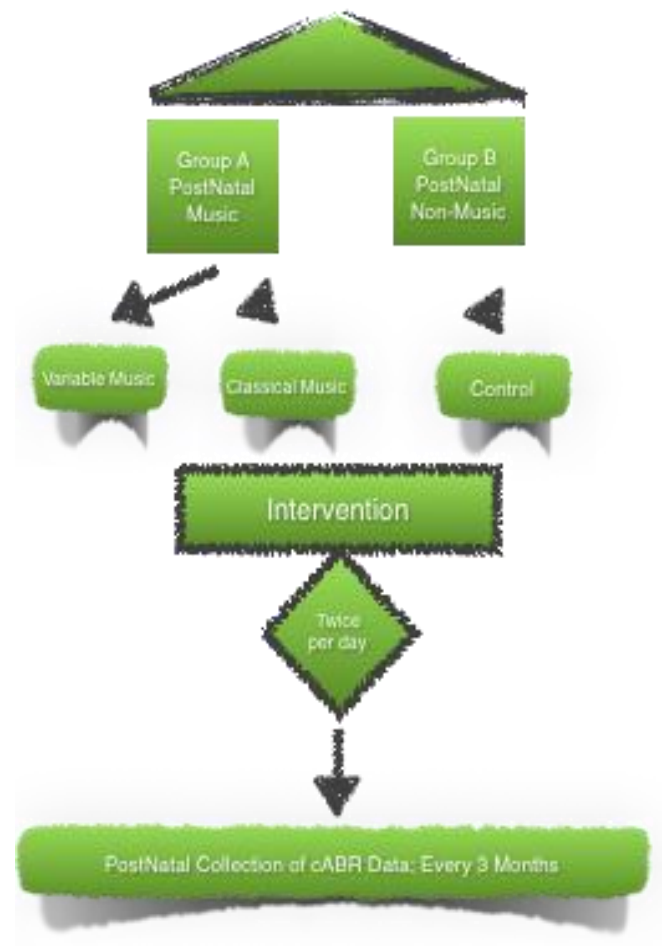

Figure 4. The research design

\section{Why use lullabies?}

The need for an early music education and enculturation in our lives has been scientifically pinpointed very early in the human history; since the $4^{\text {th }}$ century B.C. by Aristotle [34]. It is evident that the universal characteristics of the early years songs and lullabies encapsulate a dynamic prosodic structure, being able to sooth, arouse, engage and attract infants' 
attention, while acting as emotional vehicles for mothers, provide a basis to teach the native language, regulate behaviour, enhance learning and memory.

Ilari [35] has proven throughout her studies that infants in the first instances of their lives crave to come into contact with game-songs and lullabies achieving through this process to minimise their stress levels while promoting psychological bonding between them and their caregivers. Numerous studies [38] have brought to the fore lullabies' soothing function as they set up a calm and secure context for the newborn.

In that sense, singing lullabies and the "motherese" are common phenomena in most human cultures, proving the spontaneous usage of voice's musicality in basic communication [39], [40]. This early communicative dimension - both through its narrative or its singing approach - is characterised by simple structures which are constantly applied in a very expressive and ritualistic manner [41-43]. This exact presentation structure and emotional content the lullabies carry in themselves [44], [45] deem them valuable and suitable to be used in this project, where structural simplicity and emotional load can hypothetical help the auditory brainstem response analysis on the one hand, while evoke on the other hand significant neuroplastic changes we will be able to study at a great extent in a short period of experimental time.

On top of all this, we need to stress the fact that lullabies can shape an infant's musical identity. The former can shape basic life characteristics and features, like language and social engagement, hence providing a direct bridge to developing biological systems and functions.

Evidence coming from extended studies conducted by Colwyn Trevarthen has shown that musicality is indeed an inherent and very fundamental human trait, equalising or even extending itself beyond the human language [46]. Trevarthen and Malloch [47] stress that this inherent musical ability constitutes a motivational power for infants to acquire language skills, as they learn through it how to recognise rhythmical and tonal motives, following an extensive interactive process with their parents. The aforementioned researchers, trying to depict how this musical ability is or could be embedded in an infant's emotional discourse with its environment, developed the theory of "communicative musicality" [48] where integrated melodic and rhythmical expressions project a path towards social empathy and sympathy when combined with movement. This in turn leads to language as a solid communicative and therefore developmental aspect [47-49].

The inherent musicality mentioned above constitutes for children, at least during infancy and the preschool years, a way to self-express their needs and thoughts in their day-to-day social interactions [50]. Children enjoy creating musical formations in order to entertain themselves or to communicate with their surrounding people, while in between their same agerange interactions they take an extra step, extending and interlarding these musical formations through dancing and body movements [51].

The most recent research study by Lum and Campbell [52] also brought into a greater perspective the inherent musical ability children of this early age project, suggesting that the former is severely employed throughout toddler's in between communication. They create and improvise on heard rhythmical and melodic motives their parents have presented to them, singing and moving based on this in sync with their peers during their play time.

All the above has been implied that lead towards a 'children's musical culture' to be evident, which by default seems to be greatly connected to biopsychological development through movement [53], [54], perceptive kinaesthetic neural decoding [55], [56] and towards a constantly acquired social feedback loop, being achieved through an extensive musical discourse with parents [57].

Nevertheless, Colwyn Trevarthen through Theodore Gracyk's [46] studies reminds us about the fact that the aforementioned musicality can unfortunately disappear after conforming to social expectancies [58]. Gracyk has noted that even if later formal musical education can indeed emphasise towards the development of children's social abilities, the early absence of it can technically harm communication and interactions. For that matter, although it seems that creative activities may exist and come into play very early in life, in reality they do not follow a well informed framework of implementation, vitiating in this way the foundations of constructiveinvestigative communicative discourse between children and parents or caregivers [59].

According to the this, we understand that early sound and music exposure, as well as employment of these early stages of "communicative musicality" traits need to be well researched and substantiated, starting already at the level of the primarily available communication and soothing tool: the lullabies. As has been strongly stated elsewhere, development and learning cannot be properly achieved unless musical interactions and games are properly documented, stated and implemented [60].

\section{Importance of the project}

While the early development of other brain functions (e.g. cortical visual function) has been thoroughly studied, "much less information is available on [early] auditory processing" [31]. Therefore, we do not really know how its maturation process affect the adult brain function and the whole life-span education and disorders [22].

Therefore, early auditory processing and brain structure alteration research evidence at the level of 
the brainstem, emanating from life-related music education exposure protocols, could provide valuable diagnostic information, introducing a new, or improving the already adjunct to this subcortical brain system clinical applications (e.g. early fitting of hearing implants improving early in the life the quality of leaving; early diagnosis of movement disorders connected to the brainstem; early diagnosis of auditory diseases etc.).

On top of that, it has been several times noted [36] that there is not just one educational method to teach and learn music. Therefore, music education starting from an early age should encompass and refer to the all learning styles and approaches possible [37] following all possible particularities of each individual child. In that sense, studied biological properties of each individual brainstem which are based on the average brainstem knowledge could provide a more fertile ground for individual advancement. This approach could be seen even more valuable when brain abnormalities or functional delays are present looking for an early introduction and improvement of intervention tools; for example in dyslexia cases.

After all, the fundamental (early) music education's mission is not and shouldn't be the basis on which someone becomes a successful professional musician, rather a balanced - both mentally and physiologically - person, being equipped with the right education on how to use and apply this universal tool of communication and emotional expression.

\section{References}

[1] Hollins Martin, C. J., (2014). A narrative literature review of the therapeutic effects of music upon childbearing women and neonates. Complementary therapies in clinical practice.

[2] Dorn, F., Wirth, L., Gorbey, S., Wege, M., Zemlin, M., Maier, R. F., \& Lemmer, B., (2014). Influence of acoustic stimulation on the circadian and ultradian rhythm of premature infants. Chronobiology international, (0), 1-13.

[3] Fritz, T. H., Ciupek, M., Kirkland, A., Ihme, K., Guha, A., Hoyer, J., \& Villringer, A., (2014). Enhanced response to music in pregnancy. Psychophysiology.

[4] Dearn, T., \& Shoemark, H., (2014). The Effect of Maternal Presence on Premature Infant Response to Recorded Music. Journal of Obstetric, Gynecologic, \& Neonatal Nursing, 43(3), 341-350.

[5] Chorna, O. D., Slaughter, J. C., Wang, L., Stark, A. R., \& Maitre, N. L., (2014). A Pacifier-Activated Music Player with Mother's Voice Improves Oral Feeding in Preterm Infants. Pediatrics, 133(3), 462-468.

[6] Amini, E., Rafiei, P., Zarei, K., Gohari, M., \& Hamidi, M., (2013). Effect of lullaby and classical music on physiologic stability of hospitalized preterm infants: A randomized trial. Journal of neonatal-perinatal medicine, 6(4), 295-301.

[7] Partanen, E., Kujala, T., Tervaniemi, M., \& Huotilainen, M., (2013). Prenatal music exposure induces long-term neural effects. PloS one, 8(10), e78946.

[8] Discenza, D., (2013). Prematures and Feeding Therapy: New Lullaby-Powered Research. Neonatal Network: The Journal of Neonatal Nursing, 32(6), 429-430.

[9] Allen, K. A., (2013). Music Therapy in the NICU: Is There Evidence to Support Integration for Procedural Support? Advances in Neonatal Care, 13(5), 349-352.

[10] Lai, H. L., Chen, C. J., Peng, T. C., Chang, F. M., Hsieh, M. L., Huang, H. Y., \& Chang, S. C., (2006). Randomized controlled trial of music during kangaroo care on maternal state anxiety and preterm infants' responses. International journal of nursing studies, 43(2), 139-146.

[11] Standley, J. M., (2002). A meta-analysis of the efficacy of music therapy for premature infants. Journal of pediatric nursing, 17(2), 107-113.

[12] Bargiel, M., (2004). Lullabies and play songs: Theoretical considerations for an early attachment music therapy intervention through parental singing for developmentally at-risk infants. In Voices: A world forum for music therapy (Vol. 4, No. 1).

[13] Pound, L., \& Harrison, C., (2002). Supporting musical development in the early years. McGraw-Hill International.

[14] Alipour, Z., Eskandari, N., Ahmari Tehran, H., Eshagh Hossaini, S. K., \& Sangi, S., (2013). Effects of music on physiological and behavioral responses of premature infants: A randomized controlled trial. Complementary therapies in clinical practice, 19(3), 128-132.

[15] Arya, R., Chansoria, M., Konanki, R., \& Tiwari, D. K., (2012). Maternal music exposure during pregnancy influences neonatal behaviour: An open-label randomized controlled trial. International journal of pediatrics, 2012.

[16] Tagore, T. (2009). Why music matters in childbirth. Midwifery today with international midwife, (89), 33.

[17] Perani, D., Saccuman, M. C., Scifo, P., Spada, D., Andreolli, G., Rovelli, R., Koelsch, S. (2010). Functional Specializations for Music Processing in the Human Newborn Brain. Proceedings of the National Academy of Sciences of the USA, 107(10), 4758-4763.

[18] Winkler, I., Háden, G. P., Ladinig, O., Sziller, I., \& Honing, H., (2009). Newborn infants detect the beat in music. Proceedings of the National Academy of Sciences, 106(7), 2468-2471.

[19] Gerry, D., Unrau, A., \& Trainor, L. J., (2012). Active Music Classes in Infancy Enhance Musical, Communicative and Social Development. Developmental Science, 15(3), 398-407.

[20] Trainor, L. J., Shahin, A., \& Roberts, L. E., (2003). Effects of Musical Training on the Auditory Cortex in 
Children. Annals New York Academy of Sciences, 999, 506-513.

[21] Werner, L. A., (2002). Infant auditory capabilities. Current Opinion in Otolaryngology \& Head and Neck Surgery, 10(5), 398-402.

[22] Graven, S. N., \& Browne, J. V., (2008). Auditory development in the fetus and infant. Newborn and Infant Nursing Reviews, 8(4), 187-193.

[23] Poch de Gratzer, D., (1999). Can Music Help to Improve Parent-Child Communication? Learning Music with Parents-An Argentine Experience. International Journal of Music Education, 34(1), 47-56.

[24] Trainor, L. J., Marie, C., Gerry, D., Whiskin, E., \& Unrau, A., (2012). Becoming musically enculturated: effects of music classes for infants on brain and behavior. Annals New York Academy of Sciences, 1252(The Neurosciences and Music IV: Learning and Memory), 129138.

[25] Byers, J. F., Waugh, W. R., \& Lowman, L. B., (2006). Sound level exposure of high-risk infants in different environmental conditions. Neonatal Network: The Journal of Neonatal Nursing, 25(1), 25-32.

[26] Janata, P., (2013). Cognitive Neuroscience of Music 7. The Oxford Handbook of Cognitive Neuroscience, Volume 1: Core Topics, 1, 111.

[27] Masterton, B. R. U. C. E., Jane, J. A., \& Diamond, I. T., (1967). Role of brainstem auditory structures in sound localization. I. Trapezoid body, superior olive, and lateral lemniscus. J Neurophysiol, 30(2), 341-359.

[28] Krumbholz, K., Schönwiesner, M., Rübsamen, R., Zilles, K., Fink, G. R., \& Von Cramon, D. Y., (2005). Hierarchical processing of sound location and motion in the human brainstem and planum temporale. European Journal of Neuroscience, 21(1), 230-238.

[29] Kandel E.R., (2000). Principles of Neural Science, Fourth Edition. New York: McGraw Hill.

[30] Hall, J. W., (2000). Development of the ear and hearing. Journal of perinatology: official journal of the California Perinatal Association, 20 (8 Pt 2), S12-20.

[31] Guzzetta, F., Conti, G., \& Mercuri, E., (2011). Auditory processing in infancy: do early abnormalities predict disorders of language and cognitive development? Developmental Medicine \& Child Neurology, 53(12), 10851090.

[32] Skoe, E., \& Kraus, N., (2010). Auditory brainstem response to complex sounds: a tutorial. Ear and hearing, 31(3), 302.

[33] Janata, P., (2013). Cognitive Neuroscience of Music 7. The Oxford Handbook of Cognitive Neuroscience, Volume 1: Core Topics, 1, 111.

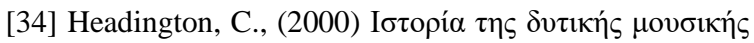

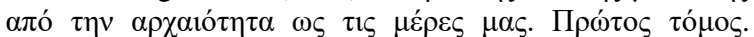

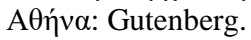

[35] Ilari, B., (2003), Singing and listening to music with infants: an interview study with Canadian mothers. Abstracts of the 5th triennial ESCOM conference,8-13 September 2003, Hanover University of Music and Drama, Germany.

[36] Lonie, D., (2010). Early Years Evidence Review: Youth Music.

[37] Schiller, P., (2010) Early brain development research review and update. Exchange, Nov/Dec. pp. 26-30.

[38] Chen-Hafteck, L., \& Mang, E., (2012). Music and language in early childhood development and learning. The Oxford Handbook of Music, 261-274.

[39] Porter, J., (2004) Lullaby. Grove Music Online. Available online at: http://www.grovemusic.com (Access Date: 20 July, 2017).

[40] Trehub, S. E., Schellenberg, E. G., (1995) Music: Its relevance to infants. Annals of Child Development, 11, 124.

[41] Trehub, S. E., (2001) Musical Predispositions in infancy. In R. J. Zatorre \& I. Peretz (Eds), The Biological Foundations of Music: Annals of the New York Academy of Sciences, 903, 1-6.

[42] Trehub, S. E., (2003a) The developmental origins of musicality. Nature Neuroscience, 6 (7), 669-673.

[43] Trehub, S. E., (2003b) Musical Predispositions in infancy: An Update. In R. J. Zatorre \& I. Peretz (Eds), The Cognitive Neuroscience of Music (pp. 3-20). Oxford: Oxford University Press.

[44] Grosléziat, C., (2012). Bébés chasseurs de sons-1001 $b b n^{\circ} 21$. Eres.

[45] Fox, D. B., (1991) Music, development, and the young child. Music Educators Journal, 77(5), 42-46.

[46] Gracyk, T., (2004) Does everyone have a musical identity? Reflections on Musical Identities. Action, Criticism, and Theory for Music Education, 3 (1), n1.

[47] Trevarthen, C. and Malloch, S., (2002), "Musicality and music before three: Human vitality and invention shared with pride", Zero to three, 23 (1), pp.10 - 18.

[48] Malloch, S. N., (1999). Mothers and infants and communicative musicality. Musicae scientiae, 3 (1_suppl), pp. 29-57.

[49] Trevarthen, C., (1999). Musicality and the intrinsic motive pulse: Evidence from human psychobiology and infant communication. Musicae scientiae, 3 (1_suppl), pp. 155-215. 
[50] Flohr, J. and Trevarthen, C., (2007). Music Learning in Childhood-Early Developments of a Musical Brain and Body, in Rauscher, F. and Gruhn, W. (eds.), Neuroscienses in music pedagogy, New York: Nova Science Publishers, pp. 53-99.

[51] Custodero, L. A., (2002). Connecting with the musical moment: Observations of flow experience in preschoolaged children. International Society for Music Education Early Childhood Conference: Children's Musical Connections, Copenhagen, Denmark.

[52] Lum, C. H. and Shehan Campbell, P., (2007). The sonic surrounds of an elementary school. Journal of Research in Music Education, 55 (1), pp. 31-47.

[53] Moorehead, G. E. and Pond, D., (1978). Music of young children, Santa Barbara, CA: Pillsbury Foundation for Advancement of Music Education.

[54] Bjørkvold, J. R., (1992). The muse within: creativity and communication, song and play from childhood through maturity. New York: Harper Collins.

[55] Peretz, I., (2001). Brain specialization for music. Annals of the New York Academy of Sciences, 930(1), 153-165.

[56] Sievers, B., Polansky, L., Casey, M., \& Wheatley, T., (2013). Music and movement share a dynamic structure that supports universal expressions of emotion. Proceedings of the National Academy of Sciences, 110(1), 70-75.

[57] Trainor, L. J., \& Heinmiller, B. M., (1998). The development of evaluative responses to music: infants prefer to listen to consonance over dissonance. Infant Behavior and Development, 21(1), 77-88.

[58] Kelly, S., (2002). A Sociological Basis for Music Education. International Journal of Music Education, 39 (1), pp. $40-49$.

[59] Prévost, E., (1995), No Sound is Innocent: AMM and the Practice of Self-Invention.

[60] Nachmanovitch, S., (2013). Creativity in life and art

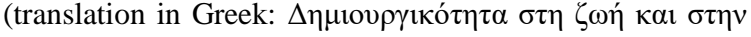

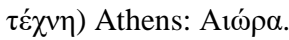

